\title{
30. Konferenz der Informationsfreiheitsbeauftragten des Bundes und der Länder in Schwerin
}

Am 30.06.2015 fand im Schweriner Schloss die 30. Konferenz der Informationsfreiheitsbeauftragten in Deutschland (IFK) statt. Unter dem Vorsitz des Landesbeauftragten für Informationsfreiheit Mecklenburg-Vorpommern, Herrn Reinhard Dankert, tagten die Informationsfreiheitsbeauftragten des Bundes und der Länder, in denen es bereits ein Informationsfreiheitsgesetz/Transparenzgesetz gibt. Das Tempo ist in den Ländern sehr unterschiedlich. Während einige Länder bereits Transparenzgesetze nach dem Vorbild Hamburgs entwickeln, gibt es in anderen Ländern noch gar keine entsprechenden Gesetze.

\section{Entschließungen}

\section{Mehr Transparenz bei den Verhandlungen über das Transatlantische Freihandelsabkommen (TTIP)!}

Noch im Jahr 2015 soll das geplante Freihandelsabkommen (TTIP) zwischen der EU und den Vereinigten Staaten von Amerika verabschiedet werden. Die Konferenz der Informationsfreiheitsbeauftragten fordert von der EU und der Bundesregierung, der Öffentlichkeit neben zusammenfassenden und erläuternden Informationen vermehrt Originaldokumente zur Verfügung zu stellen, um es den Bürgerinnen und Bürgern zu ermöglichen, sich eine eigene Meinung vom Inhalt und Ablauf der Verhandlungen zu bilden. Hierzu gehören auch Informationen über die Positionen und Forderungen der USA sowie von Lobbyisten.

Zudem setzen sich die Informationsfreiheitsbeauftragten dafür ein, dass zur Beilegung von Streitigkeiten zwischen den Handelspartnern öffentlich tagende hoheitliche Gerichte geschaffen werden. Nur dadurch kann die notwendige Transparenz gewährleistet werden.

\section{Auch Kammern sind zur Transparenz verpflichtet!}

Informationen, die im Rahmen der Tätigkeit der berufsständischen Kammern anfallen, unterfallen den Informationszugangsgesetzen des Bundes und der Länder, da die Kammern hoheitliche Aufgaben wahrnehmen. In der Vergangenheit kam es des Öfteren vor, dass Kammern der Auffassung waren, dass sie beispielsweise Informationen zu Jahresabschlüssen und Angaben zu Einnahmen, Ausgaben und Rückstellungen nicht herausgeben müssten, da es sich hierbei um „interne Informationen“ handele. Die IFK fordert daher die Kammern auf, ihren Transparenzverpflichtungen nachzukommen.

\section{Konferenz der Informationsfreiheitsbeauftragten}

Die Konferenz der Informationsfreiheitsbeauftragten in Deutschland (IFK) ist ein Gremium, das sich mit aktuellen Fragen der Informationsfreiheit beschäftigt, dass heißt mit dem Zugang zu amtlichen Informationen bei öffentlichen Stellen.

Der Konferenz der Informationsfreiheitsbeauftragten in Deutschland (IFK) gehören die Bundesbeauftragte für den Datenschutz und die Informationsfreiheit sowie die Informationsfreiheitsbeauftragten der Länder an. Bislang wurden in elf Bundesländern Informationsfreiheitsbeauftragte etabliert. Die Konferenz findet zweimal jährlich statt. Sie wird vorbereitend vom Arbeitskreis Informationsfreiheit (AKIF) unterstützt. Die Sitzungen beider Gremien sind grundsätzlich öffentlich. Die Konferenz der Informationsfreiheitsbeauftragten fasst zudem Entschließungen und Handlungsempfehlungen zu aktuellen Themen der Informationsfreiheit.

Reinhard Dankert, Der Landesbeauftragte für den Datenschutz und die Informationsfreiheit Mecklenburg-Vorpommern 